\title{
Acute Focal Bacterial Nephritis Secondary to Vesicoureteric Reflux in a Young Boy
}

\author{
Rajesh Kumar ${ }^{1}$; Devi Dayal ${ }^{1,}$; Anju Gupta ${ }^{1}$; Rakesh $\operatorname{Kumar}^{1}$; Kushaljit Singh Sodhi ${ }^{2}$; Anish \\ Bhattacharya $^{3}$ \\ ${ }_{2}^{1}$ Department of Pediatrics, Advanced Pediatrics Center, Postgraduate Institute of Medical Education and Research, Chandigarh, India \\ ${ }_{2}^{2}$ Department of Radiodiagnosis, Postgraduate Institute of Medical Education and Research, Chandigarh, India \\ ${ }^{3}$ Department of Nuclear Medicine, Postgraduate Institute of Medical Education and Research, Chandigarh, India \\ ${ }^{*}$ Corresponding author: Devi Dayal, Department of Pediatrics, Advanced Pediatrics Center, Postgraduate Institute of Medical Education and Research, Chandigarh, India. Tel:+91-1722755657, \\ Fax:+91-1722744401, E-mail: drdevidayal@gmail.com, dayal.devi@pgimer.edu.in
}

Received: December 30, 2014; Revised: February 2, 2015; Accepted: May 11, 2015

\begin{abstract}
Introduction: Acute focal bacterial nephritis (AFBN), previously called acute lobar nephronia, is a rare form of interstitial bacterial nephritis in children. The majority of patients with AFBN have an underlying urinary tract abnormality. Timely initiation of appropriate antimicrobial therapy can prevent abscess formation and help avoid the need for invasive surgical procedures.

Case Presentation:Herewe describe a case of a 4-year-old boyadmitted for a febrile urinary tractinfection(UTI). Radiological investigations led to a diagnosis of AFBN. He was later found to also have a pre-existing malformative uropathy.

Conclusions: Timely detection and appropriate antibiotic therapy of AFBN are essential to prevent renal abscess formation. A possible diagnosis of AFBN needs to be considered in all patients being treated for febrile UTI. Detection and surgical correction of the underlying malformation are necessary to prevent further recurrences.
\end{abstract}

Keywords: Nephritis; Urinary Tract Infections; Vesicoureteric Reflux; Child

\section{Introduction}

Acute focal bacterial nephritis (AFBN) is an inflammatory mass lesion caused by a localized bacterial infection, without renal abscess formation (1). The morphology of this lesion is somewhere between acute pyelonephritis (APN) and the early stages of renal abscess $(1,2)$. An underlying urinary tract abnormality, especially vesicoureteral reflux (VUR), is present more often in cases of AFBN than in children with a urinary tract infection (UTI) (1, 3-5). The pathogenesis of AFBN is similar to that of UTI and is related to either an ascending infection or hematogenous spread. However, compared to UTI, AFBN is relatively uncommon in children $(1,4)$. Pathologically, there is hyperemia, interstitial edema, and infiltration of leukocytes without necrosis or liquefaction (6). Advances in radiological imaging and an increased awareness of this condition have allowed diagnoses to be made with increasing frequency. However, diagnostic difficulties may occasionally occur due to morphological similarities with other benign or malignant renal masses, especially Wilms tumor $(7,8)$. Treatment with appropriate antibiotics is effective in all cases except occasional instances when a renal abscess develops, despite antibiotic therapy (2). Early recognition of AFBN by the treating physician is important to allow for differentiation from other renal mass lesions, to prevent its progression to a renal abscess by the timely initiation of antibiotic therapy, and to allow for examination for a surgically correctable obstructive uropathy, which is often present (2). In this communication, we report on a boy who first presented with an episode of febrile UTI and was diagnosed with AFBN and high grade VUR due to a pelviureteric junction obstruction.

\section{Case Presentation}

A 4-year-old boy was referred to our hospital with a high-grade fever and lower abdominal pain, which had persisted for 4 days. There was no dysuria or an increase in the urgency or frequency of micturition. The child had received intravenous antibiotics for 3 days prior to referral. There was no history suggestive of UTI. At presentation to our hospital, he was febrile but could tolerate oral feeding well and had normal hemodynamic parameters. His weight $(16 \mathrm{~kg},+1.2$ SDS on WHO growth charts, 2006) and height (102 cm, 0.2 SDS) were normal for his age. Results of a general physical examination were also normal. Abdominal examination revealed

Copyright (C) 2015, Pediartric Infections Research Center. This is an open-access article distributed under the terms of the Creative Commons Attribution-NonCommercial 4.0 International License (http://creativecommons.org/licenses/by-nc/4.0/) which permits copy and redistribute the material just in noncommercial usages, provided the original work is properly cited. 
left renal angle tenderness. The rest of the systemic examination was unremarkable. An initial diagnosis of febrile UTI was considered.

Results of routine hematological and biochemical investigations were normal. Urine microscopy showed only 2-4 pus cells/High Power Field. Ultrasonography revealed an enlarged left kidney $(10 \mathrm{~cm})$ with a heterogeneous echotexture and an approximately $3.4 \times 2.9$ $\mathrm{cm}$ anechoic lesion along the subcapsular location in the lower pole. The child was started on broad spectrum intravenous antibiotics (ceftriaxone, amikacin, and cloxacillin). Blood and urine cultures were sterile. Fever persisted after 4 days of intravenous antibiotics and a repeat ultrasound showed the left kidney size to be similar to the previous measurement, with multiple hypoechoic lesions in the lower pole and a minimal increase in the size of the original lesion. A contrast-enhanced computerized tomography performed on day 5 of hospitalization showed an enlarged left kidney (
$9.4 \mathrm{~cm}$ ) with a striated nephrogram pattern in the upper and lower poles (Figure $1 \mathrm{~A}, 1 \mathrm{~B}$ ). Multiple hypodense lesions (the largest, $\sim 3.0 \times 1.3 \mathrm{~cm}$ ) showing thick, irregularly enhanced walls and septations were seen clustered in the upper and lower poles, without perinephric fluid collection.

Ultrasound-guided aspiration of the lesion yielded approximately $2 \mathrm{~mL}$ of serosanguineous fluid, which was negative for both bacterial and fungal profiles based on microscopy and culture. Histopathological examination of the aspirated material showed a predominantly lymphocytic infiltrate and necrosis. No change in antibiotics was made and the child became afebrile on day 9 of hospitalization. At 2 weeks, ultrasound demonstrated a reduction in the size and normalization of the echotexture of the left kidney. Intravenous antibiotics were continued for a total of 3 weeks. A follow-up ultrasound examination at 4 weeks showed a normal-sized left kidney and no residual collection.
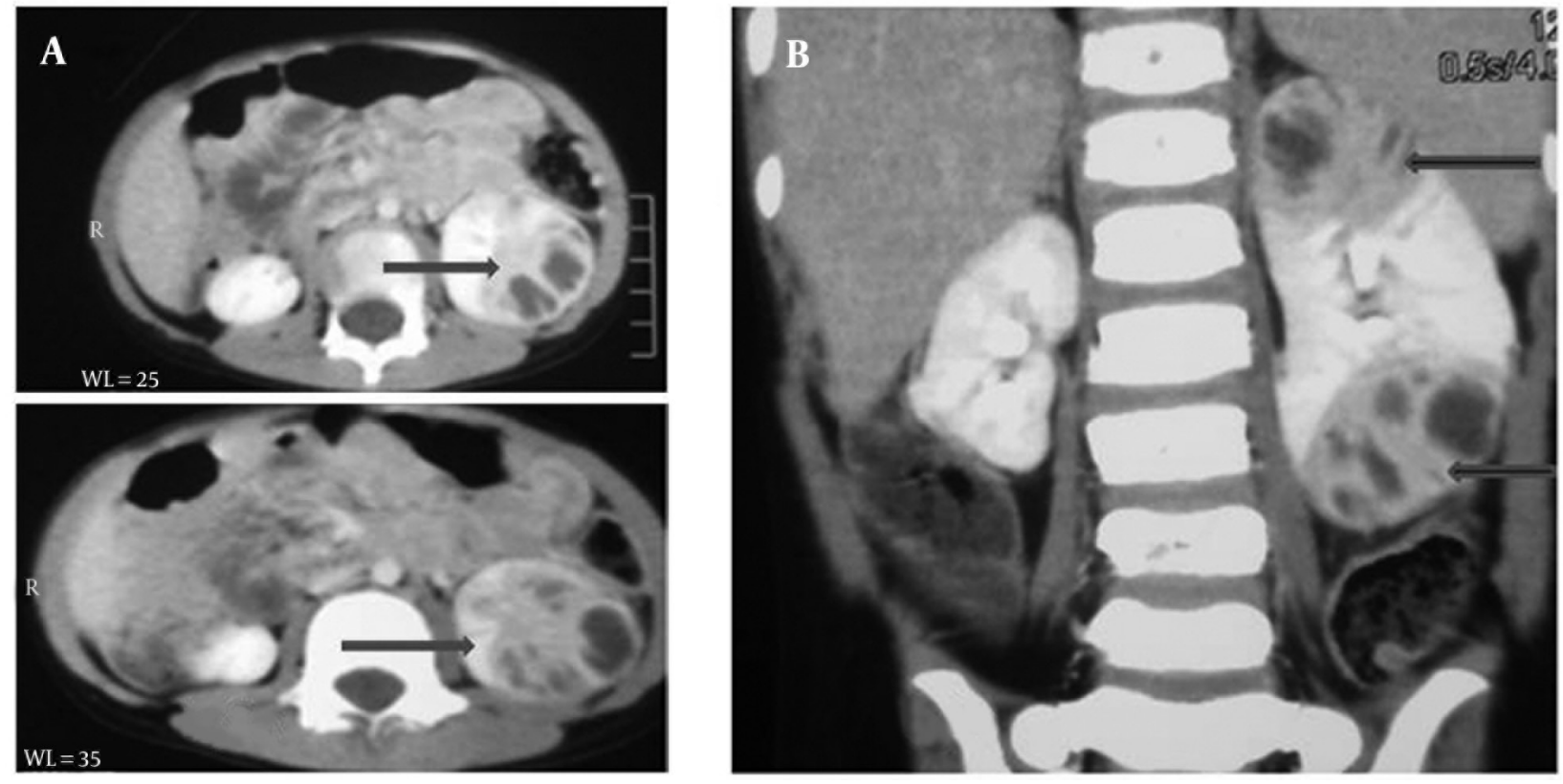

Figure 1. A and B, Contrast-enhanced Computed Tomography Showing Poorly Enhanced Lesions in the Superior and Inferior Poles of the Left Kidney

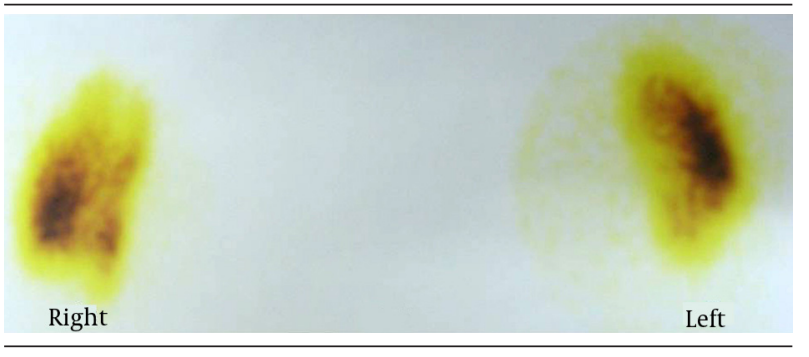

Figure 2. Dimercaptosuccinic Acid Scan Showing Poor Tracer Uptake in the Right Kidney as well as in the Superior and Inferior Poles of the Left Kidney
A dimercaptosuccinic acid (DMSA) scan performed at a 5-week follow-up visit showed negligible tracer uptake at the superior and inferior poles but an overall preserved uptake in the left kidney (Figure 2). The right kidney also showed mildly impaired cortical tracer uptake (Figure 2).

Micturating cystourethrogram showed bilateral grade 4 VUR with a grossly dilated right renal pelvis and a narrowing at the pelviureteric junction (Figure 3 ). The child underwent surgery 3 months after the AFBN episode and has remained asymptomatic over a follow-up period of 6 months. 
Kumar R et al.

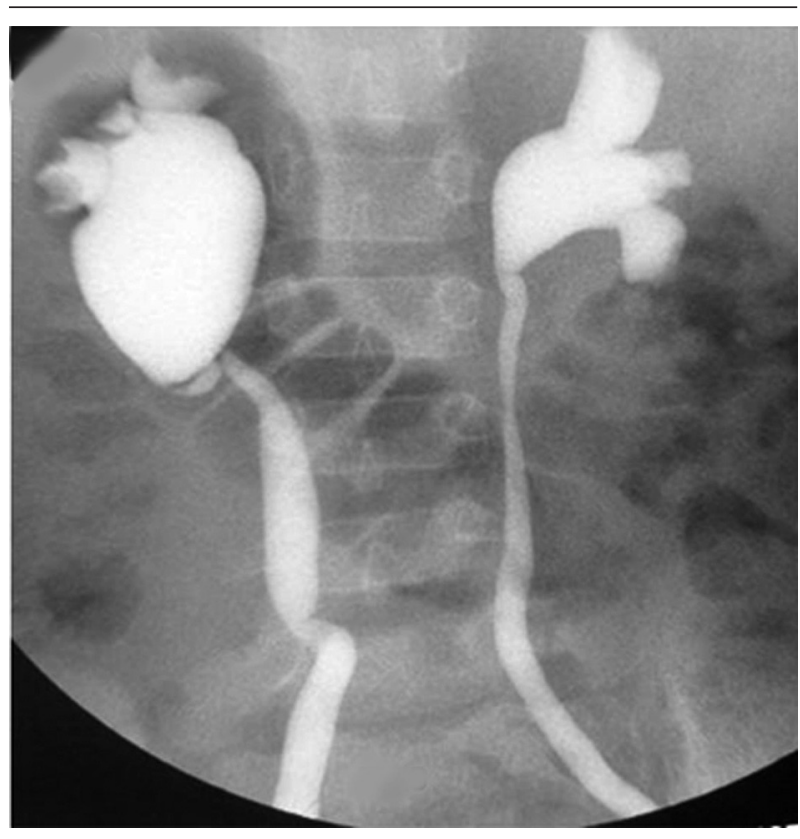

Figure 3. Micturating Cystourethrogram Showing Bilateral Grade 4 VUR with a Grossly Dilated Right Renal Pelvis and a Narrowing at the Pelviureteric Junction

\section{Discussion}

Since its first description by Rosenfield et al. AFBN has been increasingly recognized as a distinct entity that requires early recognition and the use of appropriate antibiotherapy to prevent its progression to an abscess (2, 9). Initial diagnosis may be challenging, as children can present with non-specific clinical features and radiological imaging findings may be quite misleading $(1,7,8)$. An AFBN lesion may mimic a malignancy, necessitating a percutaneous biopsy for diagnosis $(7,8)$. Additionally, in our patient, the diagnosis remained uncertain until histopathological examination of the aspirate from the kidney confirmed AFBN.

The diagnosis of AFBN is usually made based on imaging results. The characteristic ultrasonographic appearance of a focal mass with poorly defined margins in the kidney of a child with febrile UTI confirms the diagnosis in a majority of patients (10). Color Doppler ultrasound shows blood flow throughout the lesion (10). Although renal ultrasonography has been considered the best and most effective screening method, false-positive and false-negative findings may require confirmation of the diagnosis by CECT (11). Similar to that in children with UTI, the most frequently isolated causative infectious agent in AFBN is Escherichia coli, although other organisms have also been identified as the cause of infection $(3,4,12)$. No microorganisms were isolated in our patient. A probable reason may be the antibiotic therapy received by the child prior to referral to our hospital. However, negative urine cultures and a lack of leukocyturia in AFBN have been reported previously (6). In the largest study on children with AFBN, 9 out of the 80 (22\%) had sterile urine cultures (12).

The optimal duration of antibiotic therapy for AFBN is approximately 3 weeks (12). Ultrasonographic monitoring is mandatory to demonstrate the effectiveness of the antibiotic therapy in terms of the gradual reduction in the size of the lesion (12). Additionally, a follow-up DMSA scan is necessary to look for evidence of renal scarring. A high incidence of scarring has been noted in a recent study on patients with AFBN, despite adequate antibiotic treatment, similar to what is seen after recurrent UTI (13, 14). The presence of a pre-existing malformation of the urinary tract, especially VUR, as seen in our patient, also poses a significant risk for the development of AFBN and must be surgically corrected (3). However, patients with AFBN without an underlying abnormality of the urinary tract have also been reported (15). In conclusion, AFBN must be considered as a possibility in all children with febrile UTI as timely and appropriate antibiotic therapy may prevent its progression to an abscess. A longer duration of antibiotics to ensure complete resolution is recommended and surgery for any underlying malformation is necessary to prevent future recurrences.

\section{Authors' Contributions}

Rajesh Kumar: collection of data and preparation of initial manuscript draft, Devi Dayal: patient care, literature review, and preparation of the final draft, Anju Gupta: review and editing of final version of the manuscript, Rakesh Kumar: patient care and intellectual input during manuscript drafting, Kushaljit Singh Sodhi: interpretation of radiological data, Anish Bhattacharya: interpretation of nuclear scanning data.

\section{References}

1. Seidel T, Kuwertz-Broking E, Kaczmarek S, Kirschstein M, Frosch M, Bulla M, et al. Acute focal bacterial nephritis in 25 children. Pediatr Nephrol. 2007;22(11):1897-901.

2. Shimizu M, Katayama K, Kato E, Miyayama S, Sugata T, Ohta K. Evolution of acute focal bacterial nephritis into a renal abscess. Pediatr Nephrol. 2005;20(1):93-5.

3. Uehling DT, Hahnfeld LE, Scanlan KA. Urinary tract abnormalities in children with acute focal bacterial nephritis. BJU Int. 2000;85(7):885-8.

4. Abdinia B, Mohkam M, Karimi A, Alikhah F. Prevalence Rate of Urinary Tract Infection and Disorder of Urinary Profiles in the 7-12 Years Old Healthy Children in Tabriz, Iran. Arch Pediatr Infect Dis. 2013;2(2):122-5.

5. Shirvani F, Sharifian M, Mohkam M. The Value of Renal Scintigraphy With DMSA for Assessing Vesicoureteral Reflux in Children With Suspected Urinary Tract Infection. Arch Pediatr Infect Dis. 2012;1(1):27-30.

6. Montejo M, Santiago MJ, Aguirrebengoa K, Garcia B, Goicoetxea J, Martin A. Acute focal bacterial nephritis: report of four cases. Nephron. 2002;92(1):213-5.

7. Sahni VA, Ly A, Silverman SG. Usefulness of percutaneous biopsy in diagnosing benign renal masses that mimic malignancy. $A b$ dom Imaging. 2011;36(1):91-101.

8. Vijayakumar M, Prahlad N, Nandhini G, Prasad N, Muralinath S. Child with acute lobar nephronia. Indian J Nephrol. 2010;20(3):162-5

9. Rosenfield AT, Glickman MG, Taylor KJ, Crade M, Hodson J. Acute focal bacterial nephritis (acute lobar nephronia). Radiology. 
1979;132(3):553-61.

10. Cheng CH, Tsau YK, Hsu SY, Lee TL. Effective ultrasonographic predictor for the diagnosis of acute lobar nephronia. Pediatr Infect Dis J. 2004;23(1):11-4.

11. Cheng CH, Tsau YK, Chen SY, Lin TY. Clinical courses of children with acute lobar nephronia correlated with computed tomographic patterns. Pediatr Infect Dis J. 2009;28(4):300-3.

12. Cheng $\mathrm{CH}$, Tsau YK, Lin TY. Effective duration of antimicrobial therapy for the treatment of acute lobar nephronia. Pediatrics. 2006;117(1):e84-9.
13. Cheng CH, Tsau YK, Chang CJ, Chang YC, Kuo CY, Tsai IJ, et al. Acute lobar nephronia is associated with a high incidence of renal scarring in childhood urinary tract infections. Pediatr Infect Dis J. 2010;29(7):624-8.

14. Najib KH, Fallahzadeh E, Fallahzadeh MK, Fallahzadeh MH, Erjaee A. Renal scar formation in children with recurrent urinary tract infections. Iran Red Crescent Med J. 2009;2009(1):93-5.

15. Alaygut D, Bayram M, Soylu A, Turkmen M, Kavukcu S. Acute focal bacterial nephritis developed in a healthy child. Turk J Pediatr. 2013;55(2):226-8. 